\title{
Controlled diastereoselectivity at the alkene-geometry through selective encapsulation: $E-Z$ photoisomerization of oxazolidinone-functionalized enecarbamates within hydrophobic nano-cavities $\uparrow$
}

\author{
Hideaki Saito,${ }^{a b}$ J. Sivaguru, ${ }^{a}$ Steffen Jockusch, ${ }^{a}$ Joanne Dyer, ${ }^{a}$ Yoshihisa Inoue, ${ }^{b c}$ Waldemar Adam ${ }^{d}$ and \\ Nicholas J. Turro*ae
}

Received (in Cambridge, UK) 29th June 2006, Accepted 24th October 2006

First published as an Advance Article on the web 14th November 2006

DOI: $10.1039 / \mathrm{b} 609177 \mathrm{f}$

Photoisomerization of encapsulated $Z$-enecarbamates within the hydrophobic chiral cavities of $\gamma-\mathrm{CD}$ showed higher diastereoselectivities in the photoproducts than those obtained in solution. The selective encapsulation of the enecarbamates and the following isomerization process are both diastereoselectively controlled by $\gamma-\mathrm{CD}$.

Understanding the intricacies involved in photoisomerization within confined cavities has provided insights into the excited state processes that occur in biological systems. ${ }^{1}$ Supramolecular assemblies such as micelles, zeolites and cyclodextrins have been shown to be very effective in controlling the excited state processes during phototransformation. ${ }^{2}$ Immense progress has been made in recent decades to achieve high stereoselection in photoreactions in which the chiral information in the photoproducts is imprinted within the intervening short-lived excited species/reactive intermediate. ${ }^{3-6}$ Recently we have shown that oxazolidinone-functionalized enecarbamates are versatile systems for the study of conformational, electronic, stereoelectric, and steric effects. ${ }^{7-11}$ Enantioselectivity as high as 97\% was observed with these systems during photooxygenation. Further, it was clearly demonstrated that the alkene geometry is crucial to control the approach of the singlet oxygen. We have now carried out a systematic study on how to control the photoisomerization process that will provide insights into how the alkene geometry may be fine-tuned in isotropic media. ${ }^{12}$ Herein, we report the influence of cyclodextrin nano cavities ${ }^{13}$ on the diastereroselectivity of the photoisomerization process.

Diastereoselective photoisomerization of oxazolidinonefunctionalized enecarbamates 1 (Scheme 1) in solution gave low diastereoselectivities (5-15\%) upon direct and sensitized irradiation. ${ }^{12}$ Even chiral sensitizers did not significantly alter the

${ }^{a}$ Department of Chemistry, Columbia University, 3000 Broadway, Mail Code 3119, New York, NY 10027,USA.E-mail: njt3@columbia.edu ${ }^{b}$ Department of Molecular Chemistry, Osaka University, 2-1 Yamadaoka, Suita, 565-0871, Japan

${ }^{c}$ Entropy Control Project, ICORP, JST, 4-6-3 Kamishinden, Toyonaka, 560-0085, Japan

${ }^{d}$ Institut für Organische Chemie, Universität Würzburg, D-97074

Würzburg, Germany and Department of Chemistry, University of Puerto Rico, Facundo Bueso 110, Rio Piedras, PR, 00931, USA

${ }^{e}$ Department of Chemical Engineering, Columbia University, 3000 Broadway, Mail Code 3119. New York, NY 10027, USA

$\dagger$ Electronic supplementary information (ESI) available: Detailed complexation and irradiation procedures, gas chromatographic analysis, CD spectra of complex, ${ }^{1} \mathrm{H}-\mathrm{NMR}$ spectroscopy of the complex, time resolved phosphorescence measurements. See DOI: 10.1039/b609177f

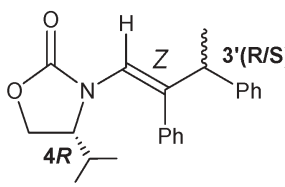

12

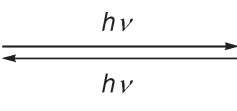

Scheme 1 Photoisomerization of 4-isopropyloxazolidinone functionalized $1^{\prime} Z-4 R\left({ }^{i} \operatorname{Pr}\right), 3^{\prime} R / S$-enecarbamates $1 Z$.

observed selectivity in these systems. ${ }^{12}$ In this context, we investigated cyclodextrin nanocavities ${ }^{13,14}$ as host to bias stereoselection during the photoisomerization processes. 4-Isopropyloxazolidinone functionalized $1^{\prime} Z-4 R\left({ }^{i} \mathrm{Pr}\right), 3^{\prime} R / S$-enecarbamates $(1 Z) \mathrm{com}$ plexed readily with $\gamma$-cyclodextrin $(\gamma-\mathrm{CD})$. The $\gamma$-CD/1 $Z$ complex was formed by adding $1 Z(0.03 \mathrm{mmol})$ in $12.5 \mathrm{~mL} \mathrm{CD} \mathrm{CD}_{3} \mathrm{OD}$ to $12.5 \mathrm{~mL} \mathrm{D} \mathrm{D}_{2} \mathrm{O}$ solution of $\gamma$-CD $(0.03 \mathrm{mmol}) .{ }^{15}$ FTIR analysis of the $\gamma-\mathrm{CD} / \mathbf{1} Z$ complex in a $1: 1 \mathrm{v} / \mathrm{v}$ of $\mathrm{D}_{2} \mathrm{O} / \mathrm{CD}_{3} \mathrm{OD}$ mixture showed the $v(\mathrm{CO})$ band of the oxazolidinone ring at $1699 \mathrm{~cm}^{-1}$ compared to that of the uncomplexed $1 Z$ in $\mathrm{CD}_{3} \mathrm{OD}$ at $1732 \mathrm{~cm}^{-1}$ (Fig. 1). The observed shift $\left[\Delta v(\mathrm{CO})=-33 \mathrm{~cm}^{-1}\right]$ strongly suggests that a host-guest complex is formed between $1 Z$ in the hydrophobic chiral cavity of the cyclodextrin; the carbonyl moiety of oxazolidinone ring is stabilized through a hydrogen bond with a proton provided by the hydrophobic inner cavity of $\gamma-\mathrm{CD} .{ }^{16}{ }^{1} \mathrm{H}-$ NMR analysis is consistent with the FTIR results, in which an

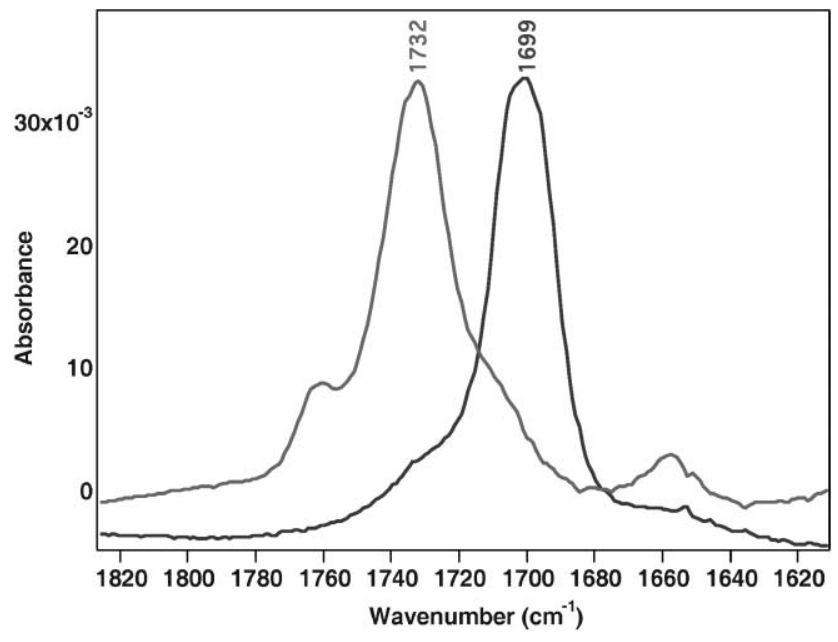

Fig. 1 FT-IR spectra of $1 Z$ in $\mathrm{CD}_{3} \mathrm{OD}$ (left) and the complex of $1 Z$ with $\gamma-\mathrm{CD}$ (right) in $1: 1 \mathrm{v} / \mathrm{v}$ of $\mathrm{D}_{2} \mathrm{O} / \mathrm{CD}_{3} \mathrm{OD}$. 


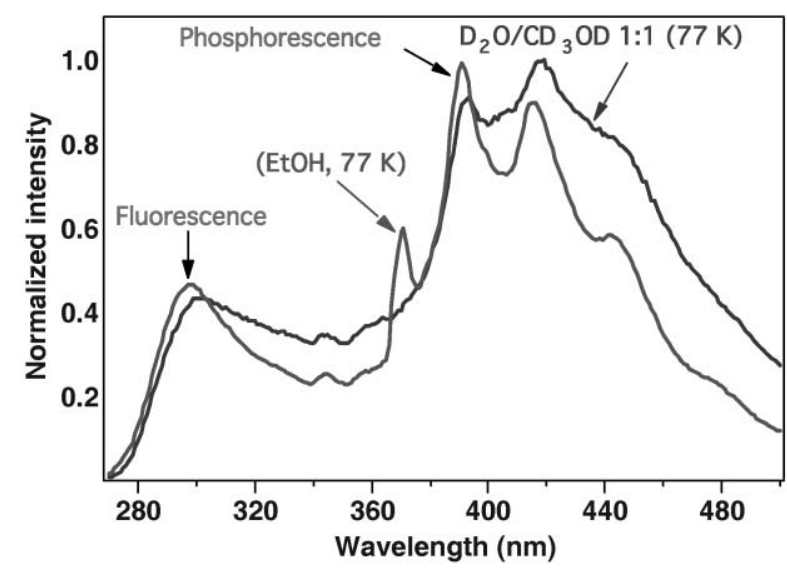

Fig. 2 Steady state emission spectra at $77 \mathrm{~K}\left(\lambda_{\mathrm{ex}}: 260 \mathrm{~nm}\right)$ of $\mathbf{1 Z}$ $\left(5.7 \times 10^{-5} \mathrm{M}\right)$ in ethanol and $\gamma-\mathrm{CD} / \mathbf{1 Z}\left([\mathbf{1 Z}]=[\gamma-\mathrm{CD}]=4.8 \times 10^{-5} \mathrm{M}\right)$ in $1: 1 \mathrm{v} / \mathrm{v} \mathrm{D}_{2} \mathrm{O} / \mathrm{CD}_{3} \mathrm{OD}$.

upfield shift in the vinylic hydrogen of the enecarbamate was observed. $^{15}$

Steady-state emission spectra of the $\gamma$-CD/1 $Z$ complex were recorded in $1: 1 \mathrm{v} / \mathrm{v}$ of $\mathrm{D}_{2} \mathrm{O} / \mathrm{CD}_{3} \mathrm{OD}$ at $77 \mathrm{~K}$, and were compared to the steady-state emission spectra of $\mathbf{1 Z}$ in an ethanol glass under identical conditions (Fig. 2). The phosphorescence emission of the $\gamma-\mathrm{CD} / \mathbf{1 Z}$ complex was broadened and slightly redshifted, which indicates that there are different orientations of the guest within the $\gamma$-CD cavity of the host. Time-resolved phosphorescence measurements ${ }^{15}$ were performed at $77 \mathrm{~K}$ by multichannel scaling using light pulses at $260 \mathrm{~nm}$ for excitation and monitoring the emission at $390 \mathrm{~nm}$. The $\gamma-\mathrm{CD} / \mathbf{1} \boldsymbol{Z}$ host-guest complex showed a phosphorescence lifetime of $\sim 27 \mathrm{~ms}$ compared to $6.6 \mathrm{~ms}$ in ethanol glass. It is clear that the increase in the lifetime was caused by encapsulation of $1 Z$ within the hydrophobic cavity of $\gamma-\mathrm{CD}$. A similar increase in lifetime upon complexation by $\gamma-\mathrm{CD}$ has previously been reported. ${ }^{17}$

Photoisomerization of the $\gamma-\mathrm{CD} / \mathbf{1} Z$ complex in $1: 1 \mathrm{v} / \mathrm{v}$ of $\mathrm{D}_{2} \mathrm{O}: \mathrm{CD}_{3} \mathrm{OD}([\gamma-\mathrm{CD}]=[1 Z]=1.4 \mathrm{mM})$ was carried out for different time intervals and the observed diasteremeric ratio (dr) in the product $E$-enecarbamates and the starting $Z$-isomer was determined both by ${ }^{1} \mathrm{H}-\mathrm{NMR}$ spectroscopy and by gas chromatography (Table 1). ${ }^{15}$ All the photoirradiations were performed on a $50: 50$ mixture of the $3^{\prime} R$ and $3^{\prime} S$ epimers for both $Z$ - and $E$-enecarbamate, unless noted otherwise. The diastereomeric ratio

Table 1 Photoisomerization of $1 Z$ with $\gamma-\mathrm{CD}$ in a $\mathrm{D}_{2} \mathrm{O} / \mathrm{CD}_{3} \mathrm{OD}$ solution

\begin{tabular}{llll}
\hline Iradiation/min $^{a}$ & $Z: E$ & $\begin{array}{l}\mathrm{dr}(\mathbf{1 Z})^{b} \\
3^{\prime} R: 3^{\prime} S\end{array}$ & $\begin{array}{l}\mathrm{dr}(\mathbf{1} \boldsymbol{E})^{c} \\
3^{\prime} R: 3^{\prime} S\end{array}$ \\
\hline 0.5 & $79: 21$ & $57: 43$ & $29: 71$ \\
1 & $67: 33$ & $56: 44$ & $39: 61$ \\
2 & $51: 49$ & $53: 47$ & $42: 58$ \\
3 & $51: 49$ & $54: 46$ & $46: 54$ \\
5 & $52: 48$ & $52: 48$ & $49: 51$
\end{tabular}

${ }^{a}[1 Z]=[\gamma-\mathrm{CD}]=1.4 \mathrm{mM}$. Photoirradiation was performed at ambient temperature in a $1: 1 \mathrm{v} / \mathrm{v}$ of $\mathrm{D}_{2} \mathrm{O} / \mathrm{CD}_{3} \mathrm{OD}$ with an excimer laser at $308 \mathrm{~nm} ; 20 \mathrm{~Hz}, 100 \mathrm{~mJ}$ pulse $^{-1}(2 \mathrm{~W})$. The diastereomeric mixture $(50: 50)$ of the starting material was used. ${ }^{b}$ Diastereomeric ratio (dr) of the starting $Z$-isomer after photoreaction. Mass balance $>95 \%$. ${ }^{c}$ Diastereomeric ratio $(\mathrm{dr})$ of the product. (dr) were enhanced by encapsulation within the hydrophobic cavity, compared to the direct irradiation of $1 Z$ without $\gamma-C D$ in $\mathrm{MeOH}$; the latter showed practically no diastereoselectivity. ${ }^{12}$ As can be seen from Table 1, high dr were observed for short irradiation ( $0.5 \mathrm{~min})$ and a decrease upon prolonged irradiation; the dr (favoring the C-3' $S$ epimer) of $\mathbf{1} \boldsymbol{E}$ decreased from $29: 71$ at $0.5 \mathrm{~min}$ to $49: 51$ at $5 \mathrm{~min}$, that is probably due to the fast isomerization of the $Z$ - to $E$-isomer. ${ }^{18-20}$

To gain more details on the origin of the selectivity observed within $\gamma-\mathrm{CD}$, the photoisomerization was investigated in the solid state. The complexation of $\gamma-\mathrm{CD}$ with $1 Z$ was achieved by adding $1 Z\left(1.5 \times 10^{-5} \mathrm{~mol}\right)$ in $5 \mathrm{~mL}$ ether and $\gamma-\mathrm{CD}\left(1.5 \times 10^{-4} \mathrm{~mol}\right)$ in $10 \mathrm{~mL}$ deionized water. A white precipitate of $\gamma-\mathrm{CD} / \mathbf{1} Z$ complex formed immediately. For easy handling of the sample, excess amount of $\gamma$-CD was used during complexation; the ratio of host : guest prepared were $10: 1$ and $20: 1$, respectively. The solid complexes were collected by filtration and washed thoroughly with ether, and the residue was dried in vacuum overnight. Diffuse reflectance Fourier transform IR spectroscopy (DRIFTS) was performed on these samples in a $\mathrm{KBr}$ matrix. The carbonyl stretch of the oxazolidinone moiety was observed at $1727 \mathrm{~cm}^{-1}$ for both $10: 1$ and $20: 1$ ratio of the $\gamma-\mathrm{CD} / \mathbf{1} Z$ complexes, compared to $1753 \mathrm{~cm}^{-1}$ for the uncomplexed $\mathbf{1 Z}$ (in $\mathrm{KBr}$ matrix). The shift $\left[\Delta v(\mathrm{CO})=-26 \mathrm{~cm}^{-1}\right]$ of the complex in the solid state was similar to that observed in a $\mathrm{D}_{2} \mathrm{O} / \mathrm{MeOD}$ solution, which indicates the formation of similar host-guest complexes in both cases.

After the extraction of the $Z$-enecarbamates from the solid complex by dissolving the samples in $\mathrm{CD}_{3} \mathrm{CN},{ }^{1} \mathrm{H}-\mathrm{NMR}$ analysis was carried out to determine the preferential diastereoselectivity of the complexation of $1 Z$ by $\gamma-C D$. Preferential complexation of the $1^{\prime} Z, 4 R\left({ }^{\prime} \operatorname{Pr}\right), 3^{\prime} S$-isomer was observed within the hydrophobic chiral cavity compared to $1^{\prime} Z, 4 R\left({ }^{i} \operatorname{Pr}\right), 3^{\prime} R$-isomer. It should be noted, therefore, that the starting $\gamma-\mathrm{CD} / \mathbf{1} Z$ complex is preferred for the C-3' $S$ epimer, with the dr of $\left(3^{\prime} R: 3^{\prime} S\right) 42: 58$ and $45: 55$ for the $20: 1$ and $10: 1$ complexes, respectively (Table 2). The solid complexes were then sandwiched between two quartz glass plates and irradiated at ambient temperature. Efficient photoisomerization was observed and the $E-3^{\prime} S$ epimer was enhanced to a dr value of $29: 71$ in the solid state (Table 2). The observed selectivity upon photoisomerization in the solid phase indicates the difference in the rate of the photoisomerization of the $Z-3^{\prime} R$ and $Z-3^{\prime} S$ epimeric enecarbamates, compared to that in $\mathrm{CD}_{3} \mathrm{OD}$ solution, for which they have similar photoisomerization rates as

Table 2 Photoisomerization of the $\gamma-\mathrm{CD} / \mathbf{1 Z}$ complex in the solid state $^{a}$

\begin{tabular}{llclll}
\hline Entry & $\begin{array}{l}\text { Mixture ratio } \\
(\gamma-C D: 1 Z)\end{array}$ & $\begin{array}{l}\text { Irradiation/ } \\
\text { min }\end{array}$ & $Z: E$ & $\begin{array}{l}\mathrm{dr}(\mathbf{1} \boldsymbol{Z})^{b} \\
3^{\prime} R: 3^{\prime} S\end{array}$ & $\begin{array}{l}\mathrm{dr}(\mathbf{1} \boldsymbol{E})^{b} \\
3^{\prime} R: 3^{\prime} S\end{array}$ \\
\hline 1 & $20: 1$ & 0 & $100: 0$ & $42: 58$ & \\
2 & & 15 & $67: 33$ & $41: 59$ & $30: 70$ \\
3 & & 30 & $60: 40$ & $40: 60$ & $32: 68$ \\
4 & $10: 1$ & 0 & $100: 0$ & $45: 55$ & $-\overline{68}$ \\
5 & & 15 & $78: 22$ & $43: 57$ & $32: 68$ \\
6 & & 30 & $66: 34$ & $43: 57$ & $35: 65$
\end{tabular}

${ }^{a}$ Irradiation was performed at $254 \mathrm{~nm}$ at ambient temperature in a Rayonet reactor. The diastereomeric mixture $(50: 50)$ of the starting material was used. ${ }^{b}$ Diastereomeric ratio (dr) of the starting $Z$-isomer after photoreaction and the product $E$-isomer. Mass balance $>95 \%$; dr was determined by ${ }^{1} \mathrm{H}$-NMR spectroscopy in $\mathrm{CD}_{3} \mathrm{CN}$ as solvent. 


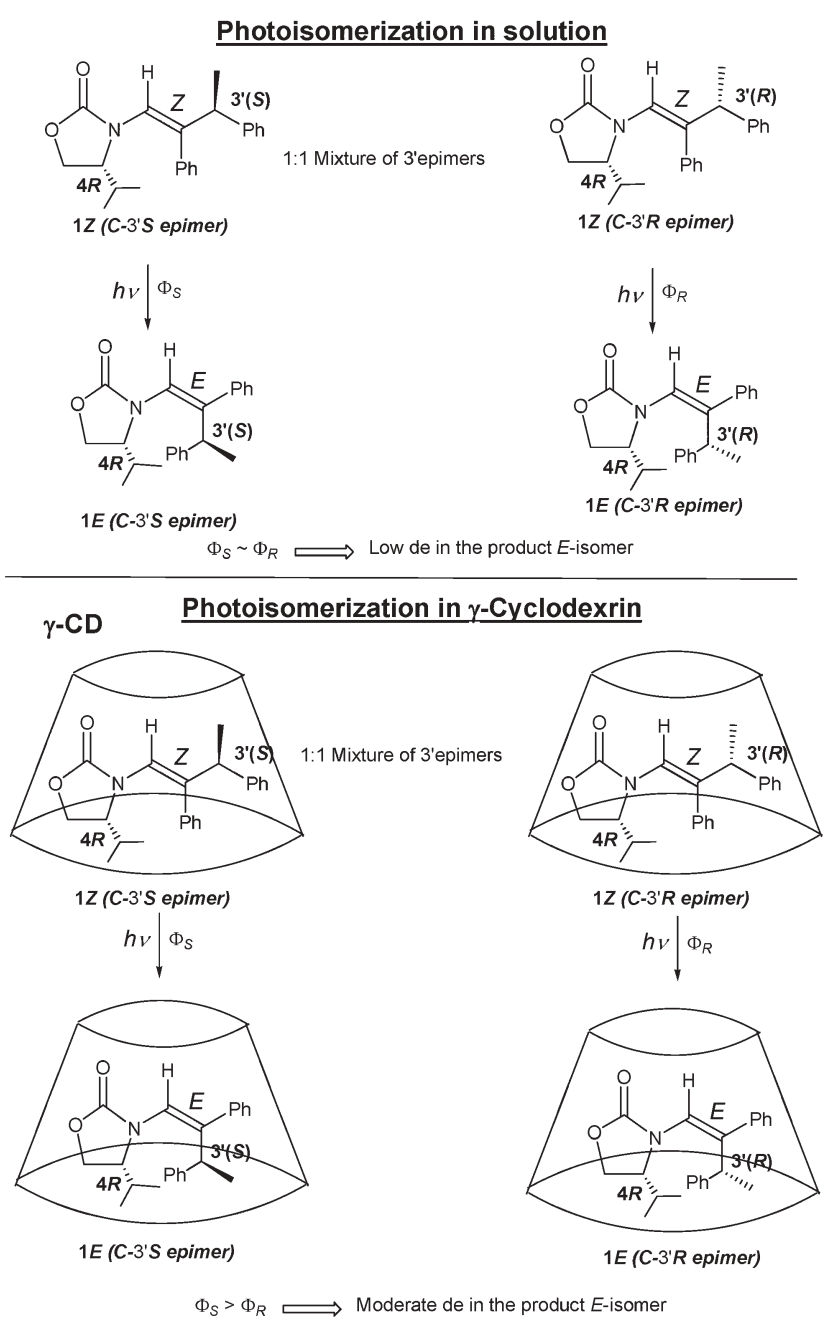

Scheme 2 Photoisomerization of 4-isopropyloxazolidinone functionalized in solution compared to that within $\gamma$-cyclodextrins.

shown by their low dr (Scheme 2-top). The photoisomerization process of $Z-3^{\prime} S$ to $E-3^{\prime} S$ epimer is clearly enhanced (Scheme 2bottom) by the complexation compared to that of the $Z-3^{\prime} R$ to the $E-3^{\prime} R$, which may be attributed to the less hindered structure of the $E-3^{\prime} S$ epimer than the $E-3^{\prime} R$ epimer in the confined cavity of $\gamma-\mathrm{CD}$.

Our current study of diastereoselective photoisomerization of oxazolidinone-functionalized enecarbamates $1 Z$ within the nano cavities of $\gamma$-CD shows that the rate of photoisomerization of the $1^{\prime} Z 4 R\left({ }^{\mathrm{i}} \mathrm{Pr}\right), 3^{\prime} R$ and $1^{\prime} Z 4 R\left({ }^{\mathrm{i}} \mathrm{Pr}\right), 3^{\prime} S$ epimers may be altered by supramolecular assemblies like cyclodextrin. The $1^{\prime} Z 4 R\left({ }^{i} \operatorname{Pr}\right), 3^{\prime} S$ epimer photoisomerizes faster than the corresponding $\mathrm{C}-3^{\prime} R$ epimer upon complexation, which presumably reflects the conformational effects in the two complexed epimers with $\gamma$-CD. This rate difference manifests itself in the observed difference in the isomerization rates that is reflected in the diastereoselectivity of the product $E$-isomer. Our current results, coupled with our photooxygenation studies, ${ }^{7-11}$ demonstrate that not only the bimolecular singlet-oxygen reaction be controlled, but also the unimolecular photoisomerization process, especially by utilizing chiral confined media such as $\gamma$-CD. Evidently, the C-3' position in the enecarbamates is critical in dictating the photoreaction within confined nano cavities. ${ }^{7-11}$

The authors at Columbia thank the NSF (CHE-01-10655, CHE-04-15516) for generous support of this research. W.A. gratefully acknowledges the financial support from the Deutsche Forschungsgemeinschaft, Alexander von Humboldt-Stiftung, and the Fonds der Chemischen Industrie. H.S. gratefully acknowledges a JSPS research fellowship (No. 08384) for young scientists. This work is dedicated to Professor Manfred Christl of the University of Wuerzburg, an appreciated colleague and esteemed friend, on the occasion of his 65th birthday; we wish him much pleasure and relaxation during his oncoming retirement.

\section{Notes and references}

1 C. Dugave and L. Demange, Chem. Rev., 2003, 103, 2475; R. S. H. Liu and G. S. Hammond, Acc. Chem. Res., 2005, 38, 396.

2 N. J. Turro, Proc. Natl. Acad. Sci. U. S. A., 2005, 102, 10766.

3 Y. Inoue and V. Ramamurthy, Chiral Photochemistry, CRC Press, Boca Raton, FL, 2004

4 A. G. Griesbeck and U. J. Meierhenrich, Angew. Chem., Int. Ed., 2002, 41, 3147.

5 Y. Inoue, Chem. Rev., 1992, 92, 741.

6 J. Sivaguru, A. Natarajan, L. S. Kaanumalle, J. Shailaja, S. Uppili, A. Joy and V. Ramamurthy, Acc. Chem. Res., 2003, 36, 509.

7 W. Adam, S. G. Bosio, N. J. Turro and B. T. Wolff, J. Org. Chem., 2004, 69, 1704.

8 W. Adam, S. G. Bosio and N. J. Turro, J. Am. Chem. Soc., 2002, 124 14004 .

9 W. Adam, S. G. Bosio and N. J. Turro, J. Am. Chem. Soc., 2002, 124, 8814.

10 T. Poon, J. Sivaguru, R. Franz, S. Jockusch, C. Martinez, I. Washington, W. Adam, Y. Inoue and N. J. Turro, J. Am. Chem. Soc., 2004, 126, 10498.

11 J. Sivaguru, T. Poon, R. Franz, S. Jockusch, W. Adam and N. J. Turro, J. Am. Chem. Soc., 2004, 126, 10816.

12 H. Saito, J. Sivaguru, S. Jockusch, Y. Inoue, W. Adam and N. J. Turro, Chem. Commun., 2005, 3424.

13 R. Breslow and S. D. Dong, Chem. Rev., 1998, 98, 1997.

14 J. Szejtli, Chem. Rev., 1998, 98, 1743.

15 Refer to the electronic supplementary information.

16 K. Tanhuanpää, K. H. Cheng, K. Anttonen, J. A. Virtanen and P. Somerharju, Biophys. J., 2001, 81, 1501.

17 S. Monti, G. Koehler and G. Grabner, J. Phys. Chem., 1993, 97, 13011.

18 B. Giese, Angew. Chem., Int. Ed. Engl., 1977, 16, 125.

19 C. D. Johnson, Chem. Rev., 1975, 75, 755.

20 A. Pross, Adv. Phys. Org. Chem., 1977, 14, 69. 